\title{
ITG sideband coupling models for zonal flows
}

\author{
M. Stransky \\ Department of Earth and Space Science, Chalmers University of Technology and Euratom-VR Association, \\ 41296 Gothenburg, Sweden
}

(Received 17 February 2011; accepted 29 March 2011; published online 16 May 2011)

\begin{abstract}
Four-wave interaction model between ITG mode and zonal flow was derived using fluid equations. In this model, the zonal flow is excited non-linearly by ITG turbulence via Reynolds stress. Numerical simulations show that the system allows for a small range above the ITG threshold where the zonal flow can stabilize an unstable ITG mode, effectively increasing $\eta_{i}$ threshold, an effect which has been called the Dimits shift. However, the shift is smaller than in known cases such that in the Cyclone base. (C) 2011 American Institute of Physics. [doi:10.1063/1.3586796]
\end{abstract}

\section{INTRODUCTION}

Radial flow shear in zonal flows in tokamak devices is known to be able to suppress turbulence and form transport barriers improving energy confinement. ${ }^{1,2}$ Zonal flows arise via a self-organization phenomenon driven by low-frequency drift-type modes, in which energy is transferred to longer wavelengths by modulational instability or turbulent inverse cascade. $^{3}$

This paper models the non-linear excitation and interaction of zonal flows with ITG turbulence modes using a fourwave interaction model based on the normal mode approach. ${ }^{4}$ One mode is used to represent the zonal mode, and three other the ITG mode and its sidebands. This is different from the system chosen in the paper by Mattor and Parker $^{5}$ where the two ITG modes form a couple with the same magnitude of $k_{x}$ and $k_{y}$ only differing by opposite radial wave direction with the zonal mode having twice the value for $k_{x}$. Sources ${ }^{6-8}$ discuss different three-wave models with bifurcations, predicting Dimits shift. The different mode structure in the presented paper is used to represent the nonlinear drive of damped zonal flow from an envelope modulated on the unstable ITG mode.

The zonal mode is modeled using vorticity equation in which the mode is driven by the Reynolds stress from the ITG modes as in Refs. 9 and 10. The ITG modes are modeled using two-fluid model with nonlinear coupling to the zonal flow mode. Following sections derive equations for the corresponding modes, while the second half of the paper deals with interactions of the modes in a simplified analytical way with pump-waves and using numerical simulations.

\section{MODEL}

\section{A. Zonal flow equation}

Assuming slab geometry and writing ion velocity as a sum of ExB, polarization, and diamagnetic drift velocities, the equation for zonal flow development is derived from the ion equation of motion by taking its curl. Taking into consideration slab magnetic field geometry, assuming that $k_{\|}$and $k_{y}$ are zero for the linear zonal flow eigenmode and neglecting curl of polarization drift velocity over the curl of ExB drift velocity and assuming that pressure gradient is collinear with density gradient, one gets vorticity type equation,

$$
\frac{d}{d t}\left(\nabla_{\perp}^{2} \phi+\frac{1}{q_{i} n_{i}} \nabla_{\perp}^{2} p_{i}\right)=v \nabla_{\perp}^{2}\left(\nabla_{\perp}^{2} \phi+\frac{1}{q_{i} n_{i}} \nabla_{\perp}^{2} p_{i}\right),
$$

where $d / d t$ is the total derivative; $\phi, n_{i}$, and $p_{i}$ are the electrostatic potential, ion density, and ion pressure respectively; and $v$ is the kinematic viscosity. Ion species considered in this paper is singly charged hydrogen type, so in the following, $e$ is used for simplicity instead of $q_{i}$. At this point, the diamagnetic contribution (terms with the Laplacian of the ion pressure) is neglected; the role of the diamagnetic effects is investigated, e.g., in Ref. 11; the viscous contribution is included to provide damping of the zonal flow but not written in intermediate steps.

\section{Interacting modes}

The interacting modes were chosen, one representing the zonal flow has index 1 , and has only $\mathrm{x}$ wave-number component. Wave with index 2 is an unstable ITG mode, and indexes 3 and 4 represent ITG upper and lower sideband modes, which can be either unstable in the regime far away from ITG stability threshold, or be stabilized due to some unspecified mechanism close to the ITG threshold.

$$
\begin{array}{ll}
\mathbf{k}_{1}=\left(q_{x}, 0,0\right), & \mathbf{k}_{2}=\left(k_{2 x}, k_{2 y}, k_{\|}\right), \\
\mathbf{k}_{3}=\left(k_{2 x}+q_{x}, k_{2 y}, k_{\|}\right), & \mathbf{k}_{4}=\left(k_{2 x}-q_{x}, k_{2 y}, k_{\|}\right) .
\end{array}
$$

Assuming slow time variation for zonal flow potential, $\phi$ can be written as

$$
\phi_{1}(\mathbf{x}, t)=\Re \mathfrak{e}\left[a_{\phi 1}(t) \mathrm{e}^{i q_{x} x}\right] .
$$

For ITG modes $(\mathrm{j}=2,3$, and 4$)$, the potential is assumed to be

$$
\phi_{j}(\mathbf{x}, t)=\Re \mathfrak{e}\left[a_{\phi j}(t) \mathrm{e}^{i \mathbf{k}_{j} \cdot \mathbf{x}-i \omega_{\mathrm{r} j} t}\right] .
$$

Imaginary part of eigenfrequency (growth or damping) is included in the time dependent amplitude.

Using weakly non-linear assumption in Eq. (1) without diamagnetic effects gives the potential amplitude of the 
zonal flow; using wave-numbers from Eq. (2), neglecting damping for the time being, a coupling equation is

$$
\begin{aligned}
\frac{d}{d t} a_{\phi 1}(t)= & \frac{k_{2 y}}{2 B}\left[\left(2 k_{2 x}+q_{x}\right) a_{\phi 3}(t) a_{\phi 2}^{*}(t)\right. \\
& \left.+\left(2 k_{2 x}-q_{x}\right) a_{\phi 2}(t) a_{\phi 4}^{*}(t)\right] .
\end{aligned}
$$

\section{B. ITG mode}

Three slab ITG modes are considered, and all are modeled using fluid equations. Ion continuity equation considers advection only by the ExB drift and thanks to slab geometry, the only non-zero velocity divergence comes from the polarization drift and parallel velocity,

$$
\frac{\partial \delta n_{\mathrm{i}}}{\partial t}+\mathbf{v}_{\mathrm{E}} \cdot \nabla\left(n_{0}+\delta n_{\mathrm{i}}\right)+n_{0} \nabla \cdot \mathbf{v}_{\mathrm{pi}}+n_{0} \nabla_{\|} v_{\|}=0 .
$$

Using Boltzmann electron relation for the linear part of the equation (zonal flow mode does not obey Boltzmann relation, so density is kept in the nonlinear interaction term), considering only linear part of polarization drift, one gets

$$
\frac{e}{T_{\mathrm{e}}} \frac{\partial \phi}{\partial t}+\frac{e}{T_{\mathrm{e}}} \frac{\partial \phi}{\partial y} v_{* \mathrm{e}}-\rho_{\mathrm{s}}^{2} \frac{e}{T_{\mathrm{e}}} \frac{\partial}{\partial t} \nabla^{2} \phi+c_{\mathrm{s}} \nabla_{\|} \frac{v_{\|}}{c_{\mathrm{s}}}=-\mathbf{v}_{\mathrm{E}} \cdot \nabla \frac{\delta n_{\mathrm{i}}}{n_{0}},
$$

where $c_{\mathrm{s}}=\sqrt{T_{\mathrm{e}} / m_{\mathrm{i}}}$ is the ion acoustic speed and $\rho_{\mathrm{s}}$ is the ion Larmor radius calculated from $c_{\mathrm{s}}$. The parallel equation of motion with ExB advection is

$$
n_{0} m_{\mathrm{i}}\left(\frac{\partial v_{\|}}{\partial t}+\mathbf{v}_{\mathrm{E}} \cdot \nabla v_{\|}\right)+\nabla_{\|}\left(n_{0} \delta T_{\mathrm{i}}+T_{\mathrm{i}} \delta n_{\mathrm{i}}\right)+e n_{0} \nabla_{\|} \phi=0 .
$$

Using Boltzmann electron relation and defining temperature ratio $\tau=T_{\mathrm{e}} / T_{\mathrm{i}}$, the following is obtained:

$$
\frac{\partial v_{\|}}{\partial t}+\frac{c_{\mathrm{s}}^{2}}{\tau} \nabla_{\|}\left[\frac{\delta T_{\mathrm{i}}}{T_{\mathrm{i}}}+(1+\tau) \frac{e \phi}{T_{\mathrm{e}}}\right]=-\mathbf{v}_{\mathrm{E}} \cdot \nabla v_{\|} .
$$

Diffusive heat is neglected in the ion energy equation, and as in the continuity equation because of the slab geometry assumption, the only non-zero velocity divergence comes from the polarization drift and parallel velocity

$$
\frac{\partial \delta T_{\mathrm{i}}}{\partial t}+\mathbf{v}_{\mathrm{E}} \cdot \nabla\left(T_{\mathrm{i}}+\delta T_{\mathrm{i}}\right)+(\Gamma-1) T_{\mathrm{i}}\left(\nabla \cdot \mathbf{v}_{\mathrm{pi}}+\nabla_{\|} v_{\|}\right)=0,
$$

where $\Gamma$ is the adiabatic ratio (5/3 is used in this paper),

$$
\begin{gathered}
\frac{1}{T_{\mathrm{i}}} \frac{\partial \delta T_{\mathrm{i}}}{\partial t}+\eta_{\mathrm{i}} \frac{e}{T_{\mathrm{e}}} \frac{\partial \phi}{\partial y} v_{* \mathrm{e}}-(\Gamma-1) \rho_{\mathrm{s}}^{2} \frac{e}{T_{\mathrm{e}}} \frac{\partial}{\partial t} \nabla^{2} \phi \\
+c_{\mathrm{s}}(\Gamma-1) \nabla_{\|} \frac{v_{\|}}{c_{\mathrm{s}}}=-\frac{1}{T_{\mathrm{i}}} \mathbf{v}_{\mathrm{E}} \cdot \nabla \delta T_{\mathrm{i}},
\end{gathered}
$$

where $\eta_{\mathrm{i}}=L_{n} / L_{T_{\mathrm{i}}}$.

To study non-linear coupling, normal mode method ${ }^{4}$ is used. The normal modes for ITG modes $(j=2,3$, and 4$)$ are

$$
a_{j}(\mathbf{x}, t)=\left[\frac{\omega_{\mathrm{L} j}}{k_{\|} c_{\mathrm{s}}}-\frac{k_{\|} c_{\mathrm{s}}(\Gamma-1)}{\tau \omega_{\mathrm{L} j}}\right] \frac{e \phi_{j}}{T_{\mathrm{e}}}+\frac{v_{\|}}{c_{\mathrm{s}}}+\frac{k_{\|} c_{\mathrm{s}}}{\tau \omega_{\mathrm{L} j}} \frac{\delta T_{\mathrm{i} j}}{T_{\mathrm{i}}} .
$$

These normal modes are subject to the nonlinear evolution equation, where the right hand side consists of matched nonlinear combination corresponding to the mode $j$ on the left hand side,

$$
\begin{aligned}
\Re \mathrm{e} & {\left[\frac{\partial}{\partial t} a_{j}(\mathbf{x} t)+i \omega_{\mathrm{L} j} a_{j}(\mathbf{x} t)\right]=-\left[\frac{\omega_{\mathrm{L} j}}{k_{\|} c_{\mathrm{s}}}-\frac{k_{\|} c_{\mathrm{S}}(\Gamma-1)}{\tau \omega_{\mathrm{L} j}}\right] } \\
& \times\left(\mathbf{v}_{\mathrm{E}} \cdot \nabla \frac{e \phi}{T_{\mathrm{e}}}\right)_{\mathrm{L}_{j}}-\left(\mathbf{v}_{\mathrm{E}} \cdot \nabla \frac{v_{\|}}{c_{\mathrm{s}}}\right)_{\mathrm{L}_{j}}-\frac{k_{\|} c_{\mathrm{s}}}{\tau \omega_{\mathrm{L} j}}\left(\frac{1}{T_{\mathrm{i}}} \mathbf{v}_{\mathrm{E}} \cdot \nabla \delta T_{\mathrm{i}}\right)_{\mathrm{L}_{j}} .
\end{aligned}
$$

Separating the wave behavior from time evolution in the normal modes, we can write them as

$$
a_{j}(\mathbf{x}, t)=\tilde{a}_{j}(t) \mathrm{e}^{-i \omega_{\mathrm{r} i} t+i \mathbf{k}_{j} \cdot \mathbf{x}},
$$

where the imaginary part of the linear eigenfrequency $\omega_{\mathrm{L} j}$ is included in the time varying amplitude. Assuming linear relationship between variables for each ITG eigenmode, we can write

$$
\frac{v_{\| j}}{c_{\mathrm{s}}}=C_{1 j} \frac{e \phi_{j}}{T_{\mathrm{e}}} \quad \text { and } \quad \frac{\delta T_{\mathrm{i} j}}{T_{\mathrm{i}}}=C_{2 j} \frac{e \phi_{j}}{T_{\mathrm{e}}},
$$

where one gets the values of constants from the linearized eigenvalue problem solution of Eqs. (7), (9), and (11).

Since the normal modes are just linear combinations of variables, the normal modes can be written as

$$
a_{j}(\mathbf{x}, t)=C_{0 j} \frac{e \phi_{j}}{T_{\mathrm{e}}},
$$

where the values of constants $C_{0 j}$ can be calculated from Eq. (12) using Eq. (15).

\section{Coupled four-wave system}

Defining dimensionless time-only varying amplitudes for all modes as

$$
\begin{gathered}
g_{1}(t)=\frac{e}{T_{\mathrm{e}}} a_{\phi 1}(t), \\
g_{j}(t)=\frac{1}{C_{0 j}} \tilde{a}_{j}(t),
\end{gathered}
$$

and rewriting zonal flow evolution (5) and nonlinearly matching (13) using relations (14) through (17), coupled three wave system is obtained

$$
\begin{aligned}
& \frac{d}{d t} g_{1}(t)=C_{+z} g_{3}(t) g_{2}^{*}(t)+C_{-z} g_{2}(t) g_{4}^{*}(t), \\
& \frac{d}{d t} g_{2}(t)=\gamma_{2} g_{2}(t)-C_{+i} g_{3}(t) g_{1}^{*}(t)+C_{-i} g_{4}(t) g_{1}(t), \\
& \frac{d}{d t} g_{3}(t)=-\delta_{3} g_{3}(t)+C_{+s} g_{2}(t) g_{1}(t), \\
& \frac{d}{d t} g_{4}(t)=-\delta_{4} g_{4}(t)-C_{-s} g_{2}(t) g_{1}^{*}(t),
\end{aligned}
$$




$$
\begin{array}{r}
C_{+z} \equiv 8 \chi_{\mathrm{B}} k_{2 y}\left(2 k_{2 x}+q_{x}\right), C_{-z} \equiv 8 \chi_{\mathrm{B}} k_{2 y}\left(2 k_{2 x}-q_{x}\right), \\
C_{+i} \equiv \frac{8 \chi_{\mathrm{B}}}{C_{02}}\left[\frac{\omega_{\mathrm{L} 2}}{k_{\|} c_{\mathrm{s}}}-\frac{k_{\|} c_{\mathrm{s}}(\Gamma-1)}{\tau \omega_{\mathrm{L} 2}}+C_{13}+\frac{k_{\|} c_{\mathrm{s}}}{\tau \omega_{\mathrm{L} 2}} C_{23}\right] k_{2 y} q_{x}, \\
C_{-i} \equiv \frac{8 \chi_{\mathrm{B}}}{C_{02}}\left[\frac{\omega_{\mathrm{L} 2}}{k_{\|} c_{\mathrm{s}}}-\frac{k_{\|} c_{\mathrm{s}}(\Gamma-1)}{\tau \omega_{\mathrm{L} 2}}+C_{14}+\frac{k_{\|} c_{\mathrm{s}}}{\tau \omega_{\mathrm{L} 2}} C_{24}\right] k_{2 y} q_{x}, \\
C_{+s} \equiv \frac{8 \chi_{\mathrm{B}}}{C_{03}}\left[\frac{\omega_{\mathrm{L} 3}}{k_{\|} c_{\mathrm{s}}}-\frac{k_{\|} c_{\mathrm{s}}(\Gamma-1)}{\tau \omega_{\mathrm{L} 3}}+C_{12}+\frac{k_{\|} c_{\mathrm{s}}}{\tau \omega_{\mathrm{L} 3}} C_{22}\right] k_{2 y} q_{x}, \\
C_{-s} \equiv \frac{8 \chi_{\mathrm{B}}}{C_{04}}\left[\frac{\omega_{\mathrm{L} 4}}{k_{\|} c_{\mathrm{s}}}-\frac{k_{\|} c_{\mathrm{S}}(\Gamma-1)}{\tau \omega_{\mathrm{L} 4}}+C_{12}+\frac{k_{\|} c_{\mathrm{S}}}{\tau \omega_{\mathrm{L} 4}} C_{22}\right] k_{2 y} q_{x},
\end{array}
$$

where $\chi_{\mathrm{B}}$ is the Bohm diffusion coefficient defined as $T_{\mathrm{e}} /$ $16 e B$. The second mode is an unstable ITG mode with linear growth-rate $\gamma_{2}$; whereas the sideband modes are written as being stable, with damping coefficients $\delta_{3}$ and $\delta_{4}$; however, they will become unstable away from the marginal instability regime. Viscous damping for the zonal flow mode (1) can be reintroduced by including damping term $-\delta_{1} g_{1}(t)$ on the right hand side of the first equation of (18) where $\delta_{1}$ can be written as $\frac{1}{2} v q_{x}^{2}$.

For the given ITG model and considered wave modes, the last four coupling coefficients from $C_{+i}$ to $C_{-s}$ simplify to

$$
C_{+i}=C_{-i}=C_{+s}=C_{-s}=8 \chi_{\mathrm{B}} k_{2 y} q_{x} .
$$

It should be noted that this simplification takes place in part because of inclusion of all three $\mathbf{v}_{\mathrm{E}} \cdot \nabla$ convective nonlinearities in the ITG model. From the three nonlinearities in this model, the parallel velocity convection term is the one necessary to get this four-wave model with damped sideband modes to exhibit zonal mode growth and ITG suppression. The other two nonlinearities alone give opposite sign to these two coupling coefficients, causing damping of the zonal mode by the growing ITG mode.

The sign of the product of $k_{2 y}$ and $q_{x}$ is unimportant because it appears in all coupling coefficients and does not change the behavior of the differential equation system. The sign of all coupling coefficients can without a loss of generality be assumed positive.

\section{SYSTEM BEHAVIOR}

The four wave system described by Eq. (18) is non-linear. However, by fixing one of the amplitudes, it becomes linear and easy to solve using traditional linear methods. By fixing different variables, one gets models which might have some physical significance; e.g., by fixing zonal flow amplitude, one can model a system with forced zonal flow by a biasing probe. In the last section, the full non-linear system is studied.

\section{A. Unstable ITG as a pump}

One way is to consider the unstable ITG mode 2 as a pump wave of constant value, so we get a linear system

$$
\begin{aligned}
& \frac{d}{d t} g_{1}(t)=-\delta_{1} g_{1}(t)+C_{+z} g_{2}^{*} g_{3}(t)+C_{-z} g_{2} g_{4}^{*}(t), \\
& \frac{d}{d t} g_{3}(t)=-\delta_{3} g_{3}(t)+C_{+s} g_{2} g_{1}(t), \\
& \frac{d}{d t} g_{4}^{*}(t)=-\delta_{4} g_{4}^{*}(t)-C_{-s} g_{2}^{*} g_{1}(t),
\end{aligned}
$$

where $g_{2}$ is now a constant. Assuming that the damping coefficients on the sidebands are the same and using equality (20), we get that this system is unstable when the amplitude of $g_{2}$ is greater than a threshold,

$$
\left|g_{2}\right|>\sqrt{\frac{\delta_{1} \delta_{3}}{C_{+s}\left(C_{+z}-C_{-z}\right)}} .
$$

Basically, this means that the zonal flow mode will not grow but will be damped until the ITG pump has reached certain threshold amplitude. Having fixed the pump wave amplitude to a value above the threshold and since the matrix coefficients are not diagonal, the modes 1,2, and 3 will eventually grow exponentially to unlimited values because this reduced system is linear. For small amplitudes, the unstable ITG mode will actually also grow, so this approximation has certain drawbacks. Another thing to note is that the threshold does not directly depend on the $k_{2 x}$ for the chosen ITG model, as it gets cancelled in the denominator of the radical. It might however enter through the value of the damping coefficient for the sidebands.

This result depends on the assumption that the ITG sidebands are damped which can be assumed only very close to the marginal stability. Away from this regime, the sidebands will be nearly as unstable as the ITG mode itself, so the damping coefficients $-\delta_{3}$ and $-\delta_{4}$ would get replaced by approximately the growth-rate $+\gamma_{2}$, and the system becomes unconditionally unstable, i.e., the zonal mode will start to grow for any parameters after initial perturbation from the equilibrium, provided that the pump amplitude is non-zero.

\section{B. Fixed zonal flow amplitude}

This is an interesting subcase which has a physical significance. It is possible to induce sheared poloidal flow by a biasing probe. Studies of influence of biasing probe on turbulence have been made on both tokamaks and stellarators, see, e.g., Refs. 12-14. The system (18) then reduces to a linear system

$$
\begin{aligned}
& \frac{d}{d t} g_{2}(t)=\gamma_{2} g_{2}(t)-C_{+i} g_{1}^{*} g_{3}(t)+C_{-i} g_{1} g_{4}(t), \\
& \frac{d}{d t} g_{3}(t)=-\delta_{3} g_{3}(t)+C_{+s} g_{1} g_{2}(t) \\
& \frac{d}{d t} g_{4}(t)=-\delta_{4} g_{4}(t)-C_{-s} g_{1}^{*} g_{2}(t)
\end{aligned}
$$

We assume that the damping coefficients on the sidebands are the same and using equality (20), we get that if $\gamma_{2}$ is greater than $\delta_{3}$ then this system is always unstable. The remaining possibility with higher damping on the third and fourth modes than growth on the second presents two critical points. One is a stability threshold, when 


$$
\left|g_{1}\right|>\sqrt{\frac{\gamma_{2} \delta_{3}}{2 C_{+i} C_{+s}}},
$$

the system is stable and damped. The second interesting point is a critical point for oscillations of ITG mode and its sidebands,

$$
\left|g_{1}\right|>\frac{\gamma_{2}+\delta_{3}}{2 \sqrt{2 C_{+i} C_{+s}}} .
$$

These oscillations would be damped for the above mentioned assumption $\left(\gamma_{2}\right.$ is smaller than $\left.\delta_{3}\right)$. Above this threshold oscillations would happen even if the assumption of $\delta_{3}$ being greater than $\gamma_{2}$ was not true; however, they would grow. Again the values of the thresholds (24) and (25) do not depend directly on $k_{2 x}$ for the chosen ITG model, expect for their possible dependence through $\gamma_{2}$ and $\delta_{3}$.

Again, this result depends on the assumption that the ITG side bands are damped which can be assumed only very close to the marginal stability. Away from this regime, replacing the sideband damping coefficients $-\delta_{3}$ and $-\delta_{4}$ by approximately the growth-rate $+\gamma_{2}$, the system becomes always unstable, i.e., the ITG and the sideband modes start to grow for any parameters after initial perturbation from the equilibrium.

\section{Four mode nonlinear system}

This system has an obvious trivial stationary point $(0,0,0,0)$, but it also has another set of stationary points and a limit cycle. Considering the Jacobian matrix at the origin, it is evident that this stationary point is hyperbolic, saddle type, therefore, locally unstable regardless if one considers damping on the sideband modes or not. The ITG mode 2 would grow for a while, whereas the zonal mode would decay in a small neighborhood of the origin. The other stationary point is actually a set of points

$$
\begin{aligned}
& g_{1}=\sqrt{\frac{\gamma_{2} \delta_{3}}{2 C_{+i} C_{+s}} \mathrm{e}^{i \theta_{1}},} \\
& g_{2}=\sqrt{\frac{\delta_{1} \delta_{3}}{C_{+s}\left(C_{+z}-C_{-z}\right)}} \mathrm{e}^{i \theta_{2}}, \\
& g_{3}=\sqrt{\frac{\delta_{1} \gamma_{2}}{2 C_{+i}\left(C_{+z}-C_{-z}\right)}} \mathrm{e}^{i \theta_{3}}, \\
& g_{4}=\sqrt{\frac{\delta_{1} \gamma_{2}}{2 C_{+i}\left(C_{+z}-C_{-z}\right)}} \mathrm{e}^{i \theta_{4}},
\end{aligned}
$$

where the phases fulfill

$$
\begin{aligned}
& \theta_{3}=\theta_{1}+\theta_{2}+2 \pi n_{1}, \\
& \theta_{4}=\theta_{2}-\theta_{1}+\left(2 n_{2}+1\right) \pi,
\end{aligned}
$$

where $n$ 's are integers. Similar relations can be derived for the case with unstable sidebands. These phase relationships allow for all-real-value stationary points. For the detailed analysis of the eigenvalues of the Jacobian matrix for this stationary set, one can easily find necessary conditions the growth rate of the ITG mode must fulfill in order to stabilize the phase relationship development, but it turns out that this is not sufficient for the whole system, and for non-zero values of the growth and damping coefficients there is always an unstable eigenvalue, though it would result in a slowly growing and oscillating mode. So conclusion about this set of stationary points is that they allow only unstable equilibria; this holds for both the cases with damped and with unstable sidebands.

Even though this non-linear system does not have any true stationary point, it actually allows for a stable limit cycle with oscillations between the ITG mode and its sidebands and offset oscillations of the zonal mode. Since it was already assumed that the mode 2 was linearly unstable, and as will be shown later, up to some value of $\gamma_{2}$, it is possible to have a stable bound solution, one can explain the Dimits shift ${ }^{15}$ with this non-linear set. However, the simulated values of the shift are much smaller than expected. The limit cycle solutions are only possible in the cases with damped sidebands, which might be the case near the linear instability threshold.

Inclusion of frequency mismatch between sidebands and ITG due to FLR effects introduces phase drift into the system. The phase drift decreases nonlinear bifurcation thresholds, but for relevant physical parameters, the qualitative behavior remains the same.

\section{Reduction to three mode system}

If one considers the side bands of the ITG mode as an amplitude modulation envelope of the mode, a constraint between the two side bands can be imposed onto the system. The constraint is that the sideband functions $g_{3}$ and $g_{4}$ (not the wave-functions themselves) are complex conjugate of each other. If one assumes the same damping/growth-rate coefficient on both sidebands, the system is then reduced into a three mode one. There is however a difference between this reduced system and a three wave system considering only one of the side bands - in this reduced system, the dependence of the coupling coefficients on the $k_{2 x}$ is cancelled. It might at a first glance look like that one can make $C_{+z}$ or $C_{-z}$ zero according to Eq. (19) by setting $q_{x}$ to $\pm k_{2 x}$ and thus suppressing coupling of the zonal flow to one of the sidebands as was done in Refs. 7 and 8; however, this suppressed sideband is still present and develops through the three wave interaction between the zonal flow and the ITG and has a direct effect on the ITG mode.

Unfortunately, this cancellation of $k_{2 x}$ does not allow one to find an optimal $q_{x}$ for a given most unstable ITG mode in this boundless slab model. For such study, one would have to resort to a bounded inhomogeneous model which is not in the scope of this article.

Having reduced the system to three mode system, all known facts about bifurcations of the three wave interaction problem can be applied. Namely, assuming damped sidebands near the ITG instability threshold, the system has an attractive limit cycle (Fig. 1) for intermediate values of $\gamma_{2}$. (In the simulations, the time dimension was normalized to $\Omega_{\mathrm{i}}\left(k_{y, \max } \rho_{\mathrm{s}}\right)^{2} / 2$ where $k_{y, \text { max }}$ corresponds to the most unstable ITG mode, which was also used as the value for $k_{2 y}$ and $k_{2 x}$.) 


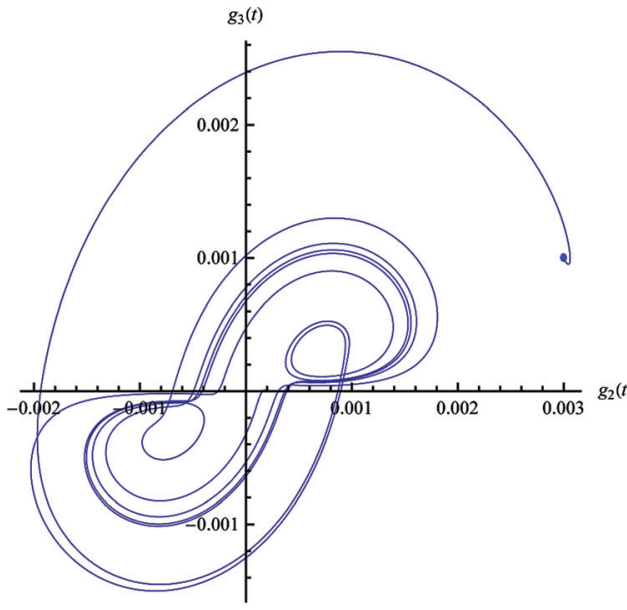

(a)

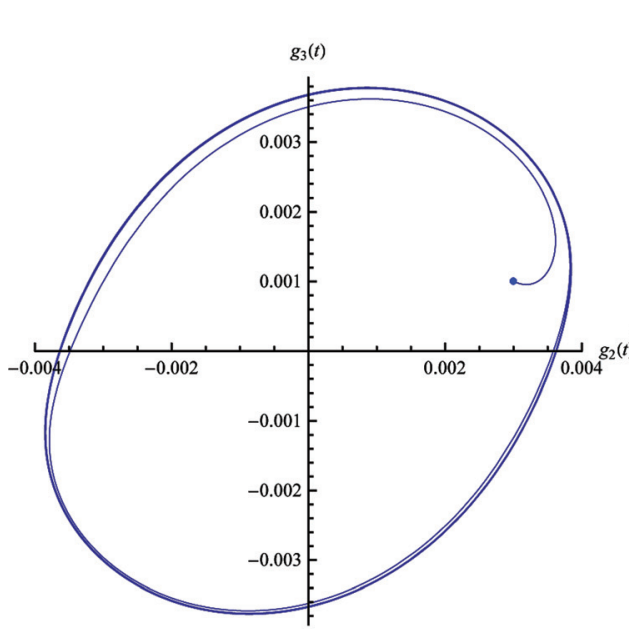

(c)

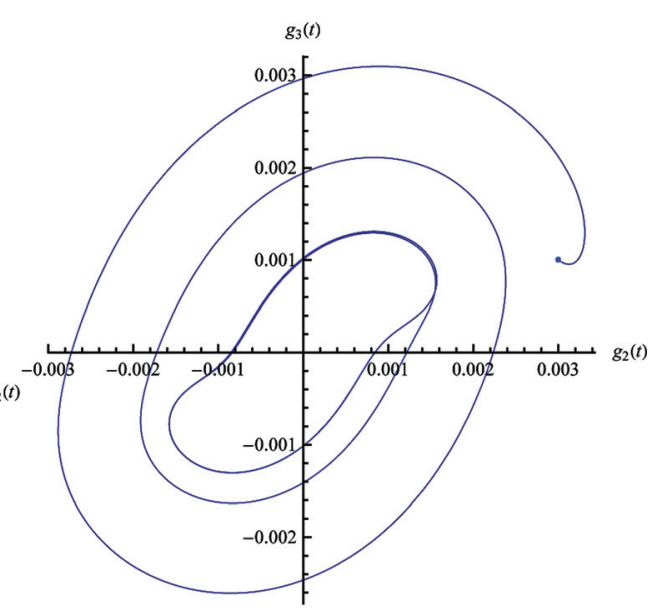

(b)

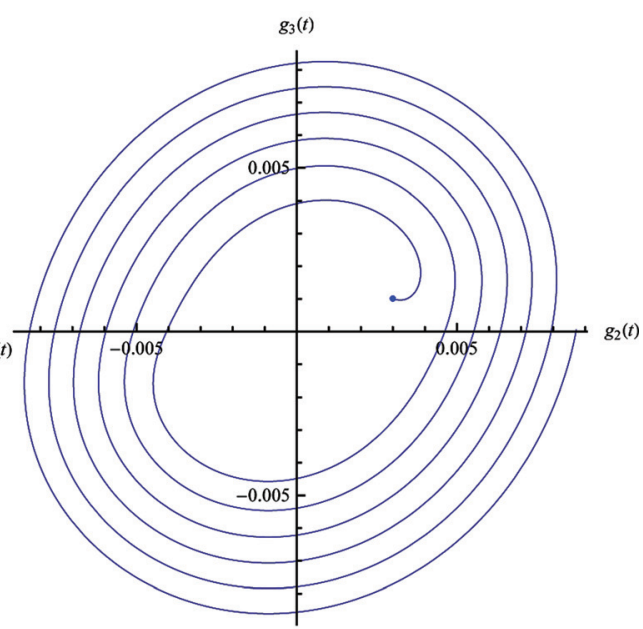

(d)
FIG. 1. (Color online) ITG amplitude $g_{3}$ vs. $g_{2}$ for (a) small $\gamma_{2}=0.00033$ with complicated behavior, (b) intermediate $\gamma_{2}=0.00077$ with simple limit cycle, (c) at the limit of stability of the system at $\gamma_{2}=0.00114$, (d) at $\gamma_{2}=0.0014$ the system is unbounded; there is no limit cycle at all. The simulations start with zero zonal flow amplitude.
This limit cycle is a fixed periodic trajectory dependent only on parameters, and not on initial conditions, providing they are in the attractive region. For values higher than a certain threshold, the system becomes unstable. There appears to be no non-linear saturation above this threshold - probably the model with just three modes is not sufficient to model such saturation above this threshold. For smaller values of $\gamma_{2}$, the limit cycle undergoes period doubling bifurcation and the behavior becomes rather complex as gamma approaches zero.

In Fig. 2, the time evolution of the zonal flow amplitude is shown for the corresponding cases in Fig. 1. The zonal flow amplitudes for cases (b) and (c) approach steady oscillations offset away from zero, not crossing it. For case (a), the zonal flow oscillation is more complex, reaching zero values. For case (d), the oscillations and the offset grow steadily.

All the graphs in Figs. 1 and 2 display the real parts of the complex variables for the four wave system reduced to the three wave one. Starting with real initial values and having no frequency mismatch, the phase development is suppressed, and the values remain real. The phase development is also suppressed if initial conditions satisfy the conditions for stationary phase (27). Having the initial conditions for the four-wave fulfill the conditions for phase (27) and amplitude relationship between sidebands, the four wave system evolves exactly the same as the reduced three wave one, as was the basis of the reduction, but it turns out that neither the initial phases nor the initial relationship between sideband amplitudes matter qualitatively and quantitatively as far as the thresholds are concerned. However, the inclusion of the phase drift due to frequency mismatch will decrease the nonlinear thresholds and ultimately change the nonlinear behavior qualitatively.

Comparing the three mode model derived from the fourwave interaction model with a simple three-wave model with only one sideband, one notices only different coupling coefficients. In the simple three-wave model, the zonal flow coupling coefficient depends on the value of the ITGs $k_{x}$, making it possible to totally suppress or change the sign, dramatically changing coupling behavior. This is not present in the four-wave model, where the dependence on the ITGs $k_{x}$ is cancelled, giving no behavior change; if one of the sideband's contribution in the zonal flow equation would be cancelled or changed its sign, the other would compensate it. Interestingly enough, the threshold mentioned in Fig. 2 does not change noticeably, assuming no pathological combination of $q_{x}$ and $k_{2 x}$ for the chosen sideband that is used.

Also to note is that the modes, in this paper most importantly the zonal mode, oscillate in all shown cases, never 


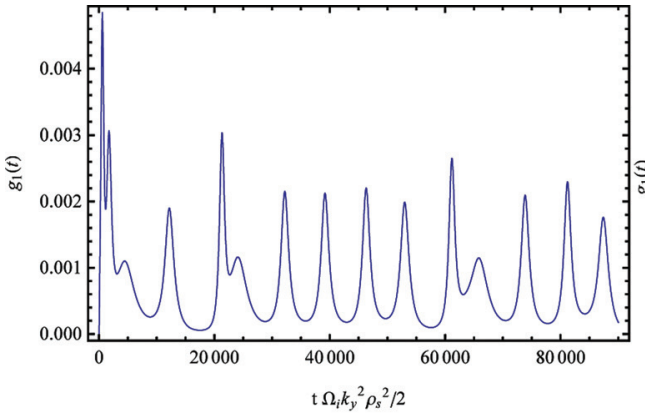

(a)

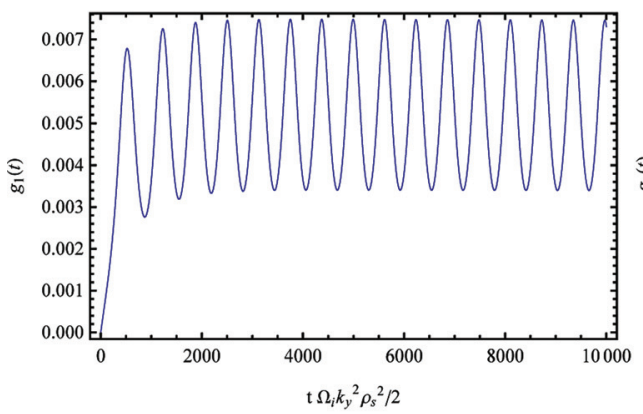

(c)

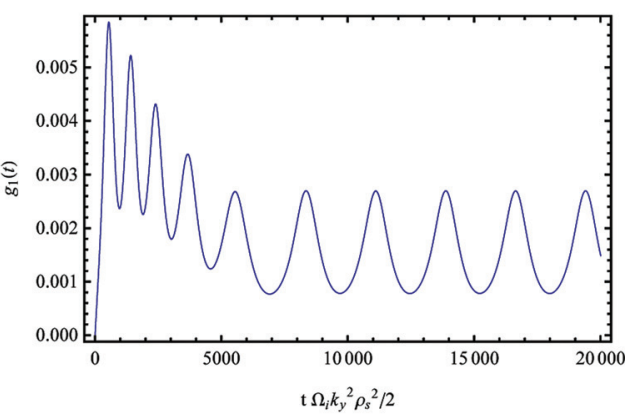

(b)

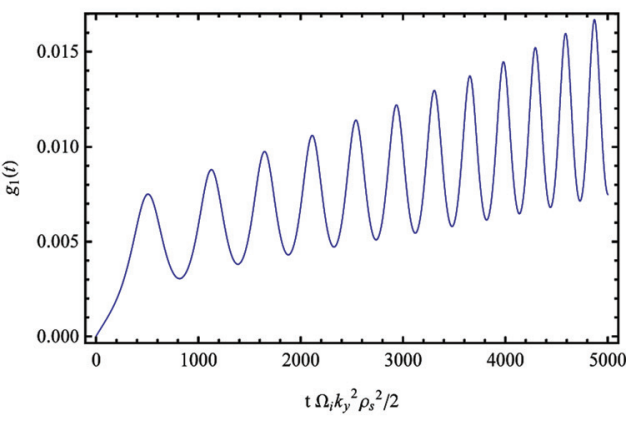

(d)
FIG. 2. (Color online) Zonal flow $g_{1}$ evolution for (a) small $\gamma_{2}=0.00033$ with complicated behavior, (b) intermediate $\gamma_{2}=0.00077$ with limit cycle, (c) at the non-linear limit of stability of the system at $\gamma_{2}=0.00114$, (d) at $\gamma_{2}$ $=0.0014$ the system is unbounded. reaching a constant value. The reason for this in this model is the lack of any stable stationary point. The Refs. 16 and 17 give numerical results linking the zonal mode oscillations to $q$ value and turbulence wave-length. Reference 7 discusses stability of a fixed point of a system which is similar to the one presented here. It should be noted that they deal with a fixed point of a one dimensional map, which in reality corresponds to an attractive limit cycle, so the result is qualitatively equivalent; however, they chose to vary the damping coefficient of the zonal mode, whereas in this paper, the main varied parameter is the ITG growth rate giving different viewpoint.

So far in this section, damping on the sidebands was assumed. This has allowed complex behavior with limit cycle possibilities. However, when instability is assumed for both the ITG and the sidebands, this is no longer possible. Assuming that the growth-rate of the sidebands is of the same order as of the ITG, the system is always unstable. The zonal flow will always oscillate, but the oscillations would be damped if the ITG growth-rate is smaller than the zonal flow damping coefficient. This behavior is not affected by the frequency mismatch apart from phase drift and beats.

The next section also assumes damped sidebands in the full four-wave model including possible frequency mismatch. It deals with cases below the non-linear threshold which was represented by (c) in Figs. 1 and 2.

\section{E. Dimits regime in the four-wave model}

The Fig. 3 shows saturated root-mean-square (RMS) value of the potential of the ITG mode in the regime between linear and non-linear thresholds. The ITG activity is not completely suppressed in this regime, but it is saturated by the four-wave interaction with the zonal flow to low amplitudes in a form of bounded limit cycles or bounded chaotic trajectories at lower values of linear ITG growth-rate as was shown above. In Fig. 3, one can see that the saturated amplitudes rapidly increase just before a non-linear stability threshold is reached when increasing $\eta_{\mathrm{i}}$. This non-linear threshold then represents the Dimits shift of the linear one. Above this nonlinear threshold, the ITG mode becomes unbounded again and other mechanism is required for saturation.

The frequency mismatch of the coupling tends to lower the thresholds for the period doubling bifurcations which lead to the chaotic trajectories at low values of the ITG growthrate. The chaotic behavior tends to increase the RMS of the ITG potential, resulting in the lower RMS amplitudes for the cases with frequency mismatch in Fig. 3 at low values of $\gamma$.

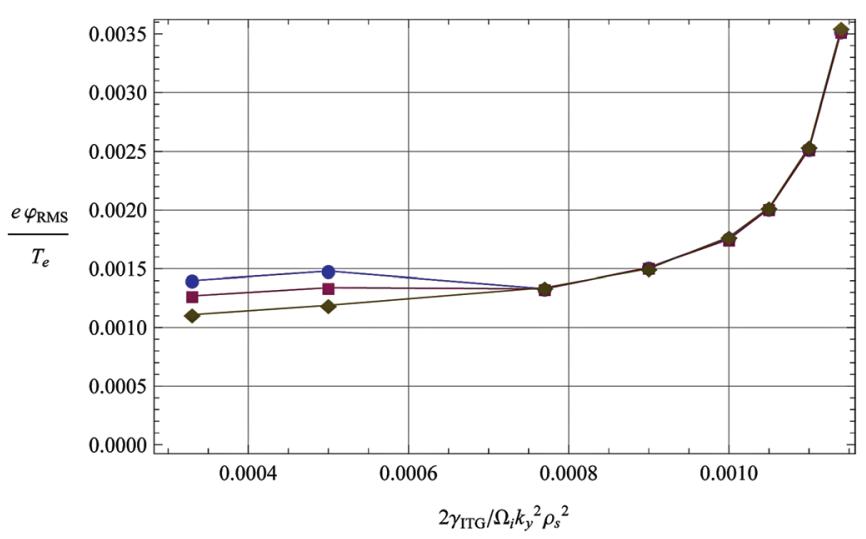

FIG. 3. (Color online) Saturated root-mean-square value of the normalized potential of the ITG mode for the four-wave system in the regime between the linear and non-linear thresholds. Circles represent points with zero frequency mismatch; squares represent points with mismatch of $0.1 \omega_{\mathrm{r}}$, and diamonds represent points with mismatch of $0.5 \omega_{\mathrm{r}}$, where $\omega_{\mathrm{r}}$ is the real part of the ITG mode frequency. 


\section{CONCLUSIONS}

The presented simple four-wave model allows for zonal mode growth through Reynolds stress, requiring a parameter dependent threshold level of ITG turbulence in the case with damped sidebands close to the ITG stability threshold. Numerical simulations also show that the system allows for a small range above the ITG threshold where zonal flow can stabilize an unstable ITG mode, effectively increasing $\eta_{\mathrm{i}}$ threshold, an effect which has been called the Dimits shift. However, the shift is smaller than in known cases such as in the Cyclone base; this is most likely due to the fact that slab ITG modes were used to model the drive mechanism but also because of uncertainty of the damping used for the zonal flows and the sideband modes near the ITG threshold. In this model in the Dimits regime, the ITG activity is not completely suppressed, but is rather saturated to low amplitudes in a form of bounded limit cycles, and the saturated amplitudes rapidly increase just before a non-linear stability threshold is reached, when increasing $\eta_{\mathrm{i}}$. This non-linear threshold then represents the Dimits shift of the linear one. Above this non-linear threshold, the ITG mode becomes unbounded again and another mechanism is required for saturation. The four-wave model is qualitatively similar to the three-wave model assuming fixed relation between the two sidebands; however, the coupling coefficients differ in magnitude, for example, the dependence of the coefficients on the value of the ITGs $k_{x}$ is cancelled in the four-wave model.

\section{ACKNOWLEDGMENTS}

The author wishes to thank Jan Weiland for the guidance and discussions that made this work possible.
${ }^{1}$ R. E. Waltz, G. D. Kerbel, and J. Milovich, Phys. Plasmas 1, 2229 (1994).

${ }^{2}$ R. E. Waltz, G. D. Kerbel, J. Milovich, and G. W. Hammett, Phys. Plasmas 2, 2408 (1995).

${ }^{3}$ P. H. Diamond, S.-I. Itoh, K. Itoh, and T. S. Hahm, Plasma Phys. Controlled Fusion 47, R35 (2005).

${ }^{4}$ W. H. Louisell, Coupled Mode and Parametric Electronics (Wiley, New York, 1960).

${ }^{5}$ N. Mattor and S. Parker, Phys. Rev. Lett. 79, 3419 (1997).

${ }^{6}$ L. Chen, Z. Lin, and R. White, Phys. Plasmas 7, 3129 (2000).

${ }^{7}$ R. A. Kolesnikov and J. A. Krommes, Phys. Rev. Lett. 94, 235002 (2005).

${ }^{8}$ R. A. Kolesnikov and J. A. Krommes, Phys. Plasmas 12, 122302 (2005).

${ }^{9}$ J. Anderson, H. Nordman, R. Singh, and J. Weiland, Phys. Plasmas 9, 4500 (2002).

${ }^{10}$ A. I. Smolyakov, P. H. Diamond, and M. Malkov, Phys. Rev. Lett. 84, 491 (2000).

${ }^{11}$ A. I. Smolyakov, P. H. Diamond, and M. V. Medvedev, Phys. Plasmas 7, 3987 (2000).

${ }^{12}$ G. Van Oost, J. Stockel, M. Hron, P. Devynck, K. Dyabilin, J. Gunn, J. Horacek, E. Martines, and M. Tendler, J. Plasma Fusion Res. Series 4, 29 (2001).

${ }^{13}$ G. Van Oost, J. Adámek, V. Antoni, P. Balan, J. A. Boedo, P. Devynck, I. Duran, L. Eliseev, J. P. Gunn, M. Hron, C. Ionita, S. Jachmich, G. S. Kirnev, E. Martines, A. Melnikov, R. Schrittwieser, C. Silva, J. Stöckel, M. Tendler, C. Varandas, M. Van Schoor, V. Vershkov, and R. R. Weynants, Plasma Phys. Controlled Fusion 45, 621 (2003).

${ }^{14}$ C. Hidalgo, M. A. Pedrosa, N. Dreval, K. J. McCarthy, L. Eliseev, M. A. Ochando, T. Estrada, I. Pastor, E. Ascasíbar, E. Calderón, A. Cappa, A. A. Chmyga, A. Fernández, B. Gonçalves, J. Herranz, J. A. Jiménez, S. M. Khrebtov, A. D. Komarov, A. S. Kozachok, L. Krupnik, A. López-Fraguas, A López-Sánchez, A. V.Melnikov, F. Medina, B. van Milligen, C. Silva, F. Tabarés, and D. Tafalla, in Proceedings of the 30th EPS Conference on Controlled Fusion Plasma Physics, St. Petersburg, Russia, July 2003, ECA Vol. 27A, P-1.21.

${ }^{15}$ A. M. Dimits, G. Bateman, M. A. Beer, B. I. Cohen, W. Dorland, G. W. Hammett, C. Kim, J. E. Kinsey, M. Kotschenreuther, A. H. Kritz, L. L. Lao, J. Mandrekas, W. M. Nevins, S. E. Parker, A. J. Redd, D. E. Shumaker, R. Sydora, and J. Weiland, Phys. Plasmas 7, 969 (2000).

${ }^{16}$ N. Miyato, Y. Kishimoto, and J. Li, Phys. Plasmas 11, 5557 (2004).

${ }^{17}$ T. S. Hahm, M. A. Beer, Z. Lin, G. W. Hammett, W. W. Lee, and W. M. Tang, Phys. Plasmas 6, 922 (1999). 